\title{
UV Raman spectroscopy of group IV nanocrystals embedded in a $\mathrm{SiO}_{2}$ matrix
}

\author{
A. C. Prieto - A. Torres $\cdot$ J. Jiménez $\cdot$ A. Rodríguez \\ J. Sangrador $\cdot$ T. Rodríguez
}

\begin{abstract}
Nanostructures of both Ge nanocrystals formed by thermal oxidation of SiGe layers, and SiGe nanocrystals formed by crystallization of amorphous SiGe nanoparticles deposited by LPCVD have been analyzed by Raman spectroscopy. The nanostructures are formed on a silicon substrate. Raman spectra have been acquired with visible $(514.5 \mathrm{~nm})$ and UV $(325 \mathrm{~nm})$ excitation lines. When the amount of material is very small, as it has happens in these nanostructures, the visible line is not able to excite the characteristic peaks of the $\mathrm{Ge}$ or $\mathrm{SiGe}$ in the Raman spectrum; instead the $\mathrm{Si}$ second order spectrum of the substrate appears and it can be misinterpreted by attributing it to the $\mathrm{Ge}-\mathrm{Ge}$ band associated with the nanocrystals. In this work, the use of UV excitation has been demonstrated to enhance the sensitivity respect to the conventional visible excitation, allowing the characteristic peaks of the $\mathrm{Ge}$ or $\mathrm{SiGe}$ nanocrystals to appear in the spectrum. We attributed this effect to the resonance effects.
\end{abstract}

\section{Introduction}

Group IV semiconductor nanocrystals embedded in a dielectric matrix have received a great interest for their potential application in Si based optoelectronics and nonvolatile memories The optical properties of $\mathrm{Si}$ and $\mathrm{Ge}$ are improved by reducing the particle size to the nanoscale. The band gap of these nanocrystals is determined by the nanocrystal size. An additional tunability tool is the use of $\mathrm{SiGe}$ alloys with varying composition. The fabrication of these nanostructures has been undertaken by means of different procedures. Among other, oxidation of polycrystalline $\mathrm{SiGe}$ layers and crystallization of amorphous SiGe nanoparticles embedded in $\mathrm{SiO}_{2}$ deposited by Low Pressure Chemical Vapour Deposition (LPCVD) are methods technologically simple and fully compatible with the CMOS technology. However, there are several technical barriers that mainly concern the control of the composition of the nanoparticles during the treatments leading to the formation of the nanocrystals. On the other hand, the crystallinity of the nanoparticles, their size and the residual stresses are factors that affect in a significant way the properties of the nanostructures. Therefore, a full characterization is necessary in order to control the main characteristics of the nanocrystals embedded in the $\mathrm{SiO}_{2}$ matrix for specific applications. Raman spectroscopy appears as a suitable tool to characterize these structures, because it is non-destructive and is sensitive to most of the structural features mentioned above

These nanostructures with nanocrystals embedded in the dielectric matrix are usually grown on a Si substrate, in this cases the Raman spectrum is a mixture of the ones of the nanostructure and the $\mathrm{Si}$ substrate; therefore, the contribution of the nanocrystals to the Raman spectrum has to be separated from the substrate contribution. In general, there is a very small amount of matter in these nanostructures; even some applications demand a few nanocrystals, which makes very difficult the detection of the Raman signal arising from them. In this case, the Raman signal of the nanocrystals can be washed out by the second order spectrum arising from the Si substrate. 
The Raman intensity depends on the Raman scattering cross section and the scattering volume, which in the case of the nanocrystals is mainly determined by the amount of material, rather than by the absorption coefficient as for bulk samples; in fact, the signal arising from the Si substrate emerges in the Raman spectra obtained on these nanostructures, which means that the stacked thickness of nanocrystals is smaller than the Raman probe depth $1 / 2 \alpha$ (with $\alpha$ the absorption coefficient at the excitation wavelength).

The use of UV excitation is demonstrated in this work to enhance the sensitivity respect to the conventional visible excitation, allowing the detection of the Raman spectrum of very small amounts of nanocrystals.

\section{Experimental procedures}

\subsection{Sample preparation}

We present herein the study of nanostructures formed by two different procedures: oxidation of SiGe layers, and thermal crystallization of amorphous SiGe nanoparticles.

Ge nanocrystals embedded in $\mathrm{SiO}_{2}$ were prepared as follows. Amorphous $\mathrm{SiGe}$ layers were deposited by LPCVD in a hot wall reactor at $425^{\circ} \mathrm{C}$. The pressure during growth was $300 \mathrm{mTorr}$ and the flow rates of the precursor gases $\left(\mathrm{Si}_{2} \mathrm{H}_{6}\right.$ and $\mathrm{GeH}_{4}$, without carrier gas) were adjusted to obtain layers with Ge fraction $x=0.3$. The substrates were silicon wafers previously oxidized to a $\mathrm{SiO}_{2}$ thickness of $750 \mathrm{~nm}$. The films were annealed at $725^{\circ} \mathrm{C}$ for $72 \mathrm{~h}$, which is a time sufficiently long to complete the crystallization. The annealing was carried out in vacuum (pressure below $60 \mathrm{mTorr}$ ) to avoid the uncontrolled oxidation of the samples; for more details see

By means of this procedure, a set of samples of continuous $50 \mathrm{~nm}$ thick amorphous SiGe layer was thermally crystallized, and subsequently oxidized in a dry oxygen atmosphere at $850^{\circ} \mathrm{C}$ for times increasing up to $7 \mathrm{~h}$. Phase separation constituted a barrier to control the particle composition, which did not keep the starting composition then becoming Ge-rich and evolving to pure Ge nanocrystals.

SiGe nanocrystals embedded in $\mathrm{SiO}_{2}$ were obtained as follows. The structures, deposited by LPCVD in a single run at $390{ }^{\circ} \mathrm{C}$, consisted of a bottom $\mathrm{SiO}_{2}$ layer deposited onto a $\mathrm{Si}(001)$ substrate, a $\mathrm{SiGe}$ layer with a $\mathrm{Ge}$ fraction of around 0.4 and a top $\mathrm{SiO}_{2}$ capping layer. $\mathrm{SiGe}$ is deposited using $\mathrm{GeH}_{4}$ and $\mathrm{Si}_{2} \mathrm{H}_{6}$ as precursor gases. $\mathrm{SiO}_{2}$ is deposited from $\mathrm{Si}_{2} \mathrm{H}_{6}$ and $\mathrm{O}_{2}$ using a flow ratio of 0.2 . Samples with SiGe layers of different thickness were deposited by adjusting the deposition time. For thickness of $5 \mathrm{~nm}$ or higher, the SiGe layers are continuous, but layers below
$5 \mathrm{~nm}$ are discontinuous giving rise to nanoparticles. Three samples have been deposited, one sample with a continuous $\mathrm{SiGe}$ layer $15 \mathrm{~nm}$ thick, other sample with a quasi continuous layer with nanoparticles of $5 \mathrm{~nm}$ diameter, and another one with around $3.5 \mathrm{~nm}$ diameter nanoparticles. The samples were annealed by rapid thermal annealing (RTA) in $\mathrm{N}_{2}$ atmosphere, at $800^{\circ} \mathrm{C}$ for $1 \mathrm{~min}$. These procedures are described elsewhere For more details about the crystallization of small nanoparticles of either Si or $\mathrm{Ge}$

\subsection{Characterization by Raman spectroscopy}

The Raman spectra were acquired with both visible (514.5 nm line from an Ar + laser) and UV (325 nm line from a HeCd laser) excitation using a HR UV Labram Jobin-Yvon Raman spectrometer. The results obtained with visible and UV excitations in thick layers were substantially identical. However, for nanostructures with very small particle size the UV excitation presented significant improvement respect to the visible excitation, as discussed later on. The Raman spectra of crystalline group IV semiconductors, $\mathrm{Si}$ and Ge, mainly consist of a first order phonon band, $520 \mathrm{~cm}^{-1}$ for Si, and $302 \mathrm{~cm}^{-1}$ for Ge. In the case of SiGe alloys first order phonon modes, arising from the vibrations associated with the different bonds in the alloy, $\mathrm{Si}-\mathrm{Si}, \mathrm{Ge}-\mathrm{Ge}$ and $\mathrm{Si}-\mathrm{Ge}$, are detected. The spectral features of these modes provide information about the chemical composition of the alloy These phonon bands are very sensitive to the crystal environment, in particular the lost of translation symmetry induces broadening and red frequency shifts, which are used to determine the crystallite size Also, strain induces a frequency shift, which is positive under compressive strain, and negative under tensile strain Besides the first order phonon bands, a series of second order (two phonon processes) bands, much weaker than the first order bands, is observed.

\section{Results and discussion}

Figure 1 presents the second order Raman spectrum of $\mathrm{Si}$ with the 2TA (transverse acoustic) band peaking at $300 \mathrm{~cm}^{-1}$, and the first order Raman spectrum of $\mathrm{Ge}$ nanocrystals of the samples obtained by thermal oxidation of SiGe layers both acquired under visible excitation. The two spectra show a strong similitude, which can induce to misleading interpretations when the amount of $\mathrm{Ge}$ nanocrystals is quite small. This problem was addressed by A.V. Kobolov , who showed the inconsistency of many Raman spectra reported as associated with Ge nanocrystals, as in reality they arise from the $\mathrm{Si}$ substrate. Furthermore, 


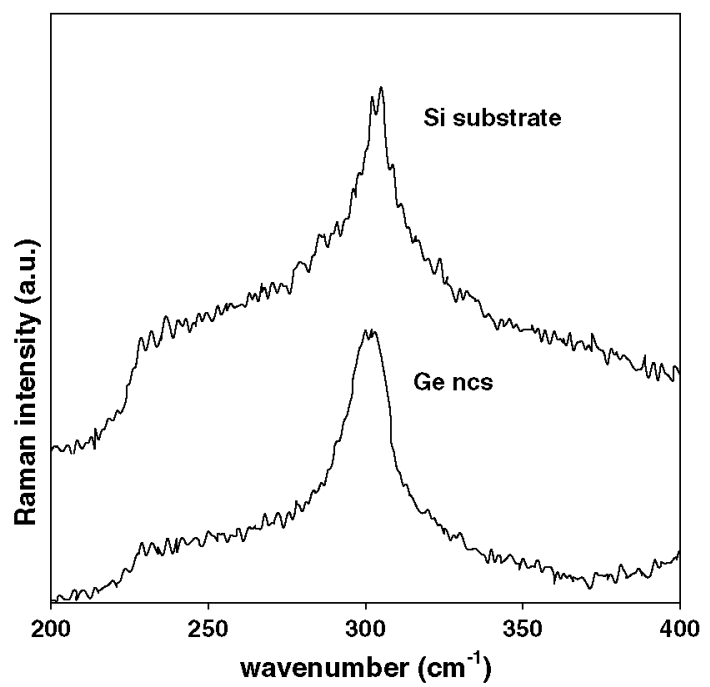

Fig. 1 First order Raman spectrum of Ge nanocrystals, and second order Raman spectrum of Si substrate. Spectra are acquired with visible excitation line $(514.5 \mathrm{~nm})$

Kolobov asked himself about the real possibility of measuring the Raman spectrum of $\mathrm{Ge}$ nanostructures on $\mathrm{Si}$ substrates. One possibility to do it is to enhance the Raman signal by resonance.

As seen in Fig. 2 the imaginary part of the dielectric function presents three resonances for $\mathrm{Ge}$ at $2.3 \mathrm{eV}, 3.2 \mathrm{eV}$ and $4.2 \mathrm{eV}$, and two resonances at $3.4 \mathrm{eV}$ and $4.2 \mathrm{eV}$ for $\mathrm{Si}$

The Raman scattering cross section is enhanced by resonance at energies close to the optical transitions for polar phonon modes. Furthermore, the absorption coefficient of Ge is strongly increased for UV excitation which also contributes to enhance the Raman signal. The above resonances are valid for bulk material. In the case of nanocrystals, quantum confinement shifts the energy of the optical transitions; therefore, one can expect resonances at

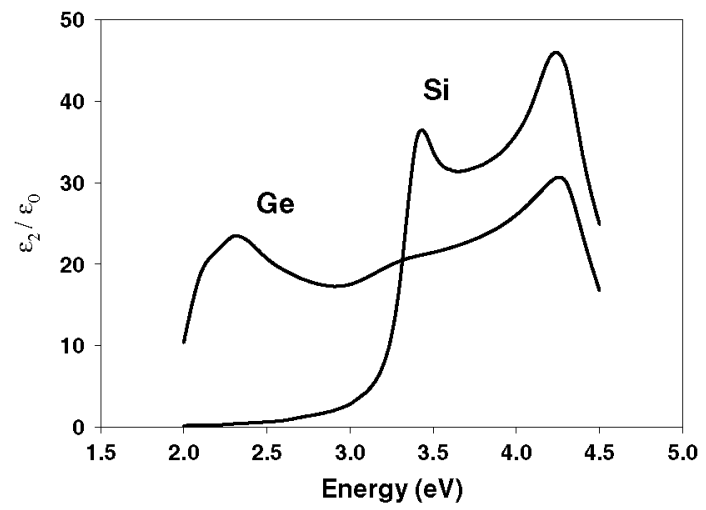

Fig. 2 Imaginary part of the dielectric function for the Ge (solid line) and $\mathrm{Si}$ (dotted line). It could be observed the same resonance in both materials at $4.2 \mathrm{eV}$. It is also induced that resonance between $2.3 \mathrm{eV}$ and $3.4 \mathrm{eV}$ and $4.2 \mathrm{eV}$ must appear for the SiGe alloy. (data from Ref. 14) different energy values. The resonance conditions should depend on the crystal size, which makes UV excitation suitable for increasing the intensity of the Raman signal of small nanocrystals.

Resonance effects under UV excitation were observed for $\mathrm{Ge}$ nanocrystals embedded in $\mathrm{SiO}_{2}$ formed by oxidation of SiGe polycrystalline layers. In this samples, once all the $\mathrm{Si}$ atoms of the SiGe layer have been oxidized a continuous thin Ge layer is formed after an oxidation time of $225 \mathrm{~min}$; further oxidation thins down the layer and forms $\mathrm{Ge}$ islands, which the size is progressively reduced by oxidation forming Ge nanocrystals of progressively smaller diameter

Figure 3 shows the Raman intensity of the Ge nanocrystals as a function of the oxidation time for both excitations $(514.5 \mathrm{~nm}$ and $325 \mathrm{~nm})$. The intensities are normalized to the intensity measured for the continuous $\mathrm{Ge}$ layer. The evolution of the Raman intensity shows the existence of a resonance for UV excitation; the Raman intensity increases for decreasing nanocrystal size. Note that indeed the Raman intensity increase should be even higher, because the volume occupied by the Ge nanocrystals is reduced for increasing oxidation time. Contrarily to the evolution of the Raman intensity for UV excitation, the Raman intensity for visible excitation does not show any significant evolution with the nanocrystal size, which is in agreement with the small confinement energy reported for the E1 excitonic transition for Ge nanocrystals with size around $5 \mathrm{~nm}$ the E1 gap scales from 2.2 to $3.3 \mathrm{eV}$ for nanocrystals with $2 \mathrm{~nm}$ diameter The average of the nanocrystals size in our samples is shown in Fig. 3. The decrease of the intensity for both excitations for long oxidation times, $315 \mathrm{~min}$, obeys the drastic reduction of the Ge volume at such an oxidation stage. The resonance associated with the UV excitation does not seem to be

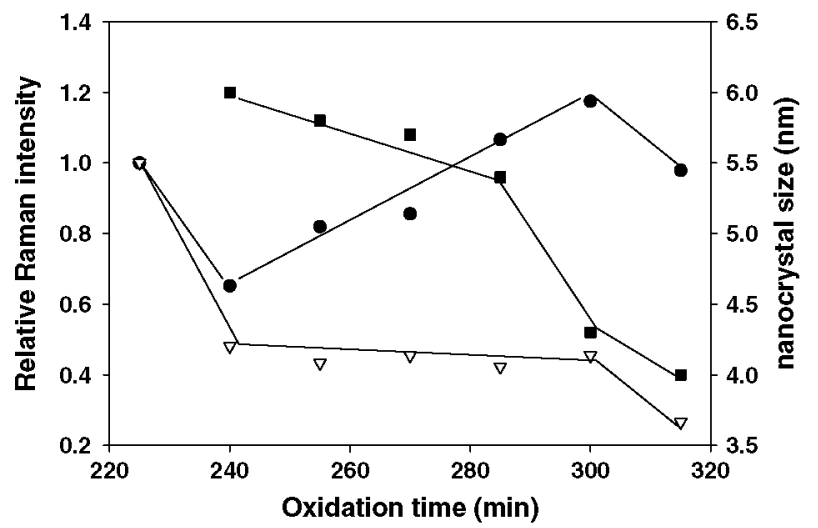

Fig. 3 Raman intensity of Ge nanocrystals obtained by oxidation of SiGe films represented as a function of the oxidation time. Data for visible excitation (triangles), and UV excitation (circles) are shown. Average size of nanocrystals (squares) is also included 


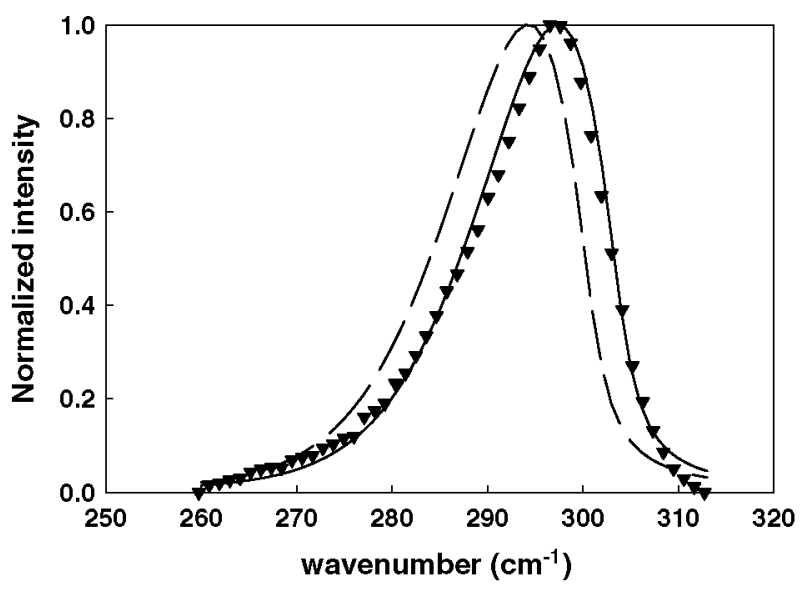

Fig. 4 Experimental Raman spectrum of Ge nanocrystals (triangles). Theoretical curve calculate using the phonon correlation length model (dashed lines). This curve is shifted to high frequencies (continuous line) when the compressive strain is taken into account, fitting the experimental data

related to E1 resonance, but it must be associated with higher energy transitions, either the zone center $\mathrm{E}_{0}{ }_{0}$ direct transition $(3.2 \mathrm{eV}$ in bulk $\mathrm{Ge})$ or the zone border $\mathrm{E}_{2}$ transition $(4.3 \mathrm{eV})$.

The average nanocrystal size was estimated by fitting the Raman spectra using a phonon correlation length model

Fig. 4. The optimal fit to the experimental data (tri-

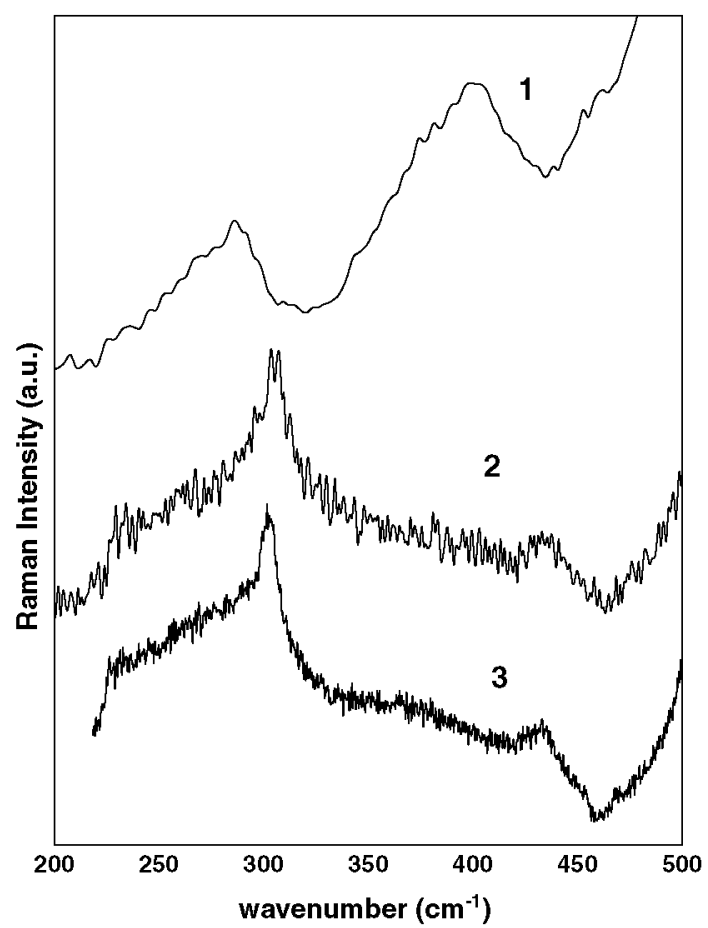

Fig. 5 Raman spectra of SiGe nanocrystals with UV (1) and visible (2) excitation lines respectively. The second order spectrum of the bare Si substrate is also drawn (3) angles) is achieved when the compressive strain is considered, solid line, while it is shifted to the low frequency respect to the experimental plot when only phonon confinement is taken into account, dashed line. A description of the fitting procedure including strain can be found in

This compressive strain supplies an additional contribution to the blue shift of the band gaps.

Figure 5 shows the Raman spectra of SiGe nanocrystals, around $3.5 \mathrm{~nm}$ in diameter, embedded in $\mathrm{SiO}_{2}$ on a $\mathrm{Si}$ substrate, obtained with either visible $(514.5 \mathrm{~nm})$ or UV $(325 \mathrm{~nm})$ excitation; the second order Raman spectrum (visible excitation) of a bare Si substrate is also shown. If one compares the visible spectrum of the SiGe nanostructure and the Si substrate spectrum, one observes that they have an one to one correspondence, evidencing that visible excitation cannot detect the small amount of SiGe nanocrystals. The spectrum of Fig. 5 obtained under UV exci-
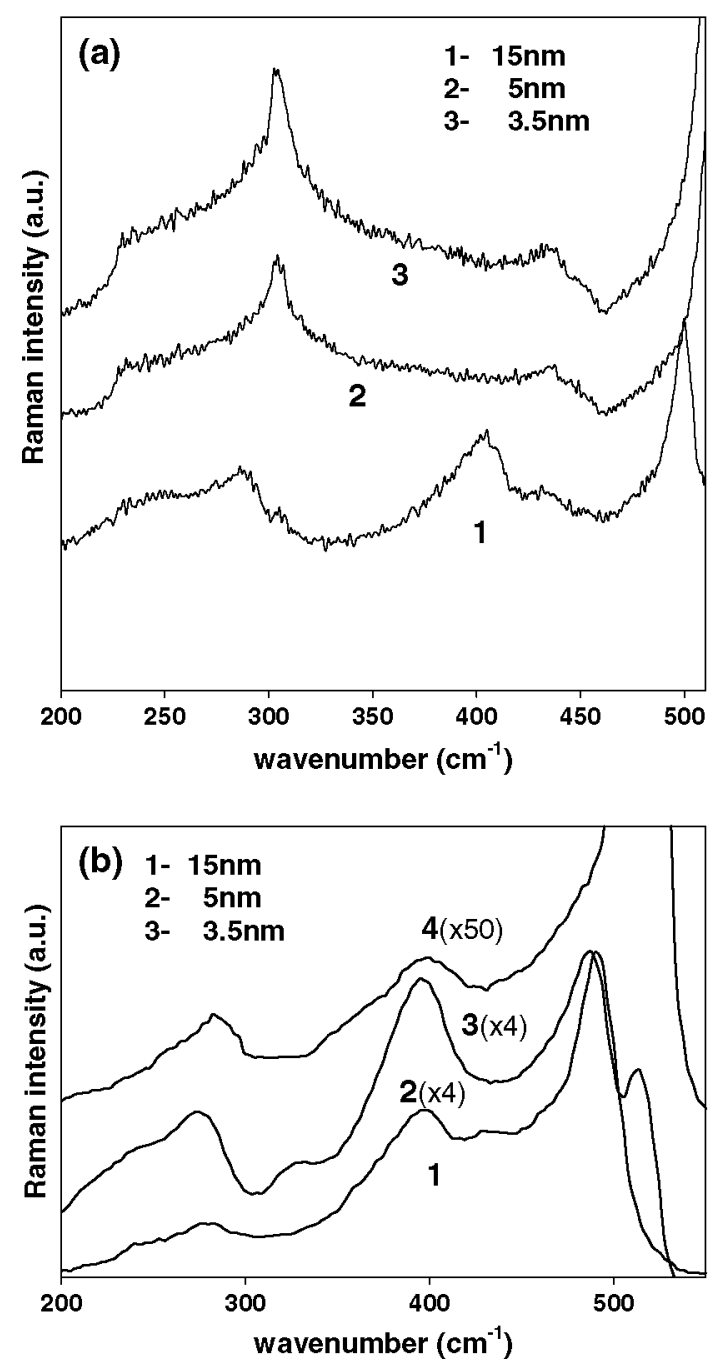

Fig. 6 Raman spectra of samples with SiGe layers of different thickness (see text). (a) Visible excitation; (b) UV excitation 
tation, clearly shows the Ge-Ge and Si-Ge bands corresponding to the presence of the SiGe nanocrystals.

Figure 6 shows the Raman spectra of the three SiGe samples with visible and UV excitation. The spectra obtained with visible excitation, Fig. 6a, reveal the presence of SiGe only for the $15 \mathrm{~nm}$ thick layer, while the spectra of the samples with nanoparticles, 5 and $3,5 \mathrm{~nm}$ in diameter, cannot reveal their presence. The only features observed correspond to the first and second order spectrum of the Si substrate. Nevertheless, the spectra obtained with UV excitation, Fig. 6b, show for the three samples the $\mathrm{Ge}-\mathrm{Ge}$ and $\mathrm{Si}-\mathrm{Ge}$ bands corresponding to the SiGe nanoparticles, suggesting that the $325 \mathrm{~nm}(3.81 \mathrm{eV})$ excitation is close to a resonance of the $\mathrm{SiGe}$ nanocrystal system. As it can be seen in Fig. 2, the SiGe resonances must appear in in a rough estimation in between 3.5 and $4.5 \mathrm{eV}$.

In summary, the use of the UV $(325 \mathrm{~nm})$ instead of visible $(514.5 \mathrm{~nm})$ excitation allows the study by Raman spectroscopy of nanostructures with a very small amount of nanocrystalline material due to the increase in sensitivity caused by the resonance effects. This effect enhances the $\mathrm{Ge}-\mathrm{Ge}$ and $\mathrm{Si}-\mathrm{Ge}$ bands of the spectrum of the nanoparticles and therefore avoids possible misleading interpretations caused by the influence of the second order spectrum of the Si substrate.

\section{References}

E.W.H. Kan, W.K. Choi, W.K. Kim, E.A. Fitzgerald, D.A. Antoniadis, J. Appl. Phys. 95, 3148 (2004)

M. Avella, A.C. Prieto, J. Jimenez, A. Rodríguez, J. Sangrador, T. Rodríguez, Solid. St. Común. 136, 224 (2005)

M.I. Ortiz, A. Rodríguez, J. Sangrador, T. Rodríguez, M. Avella, J. Jiménez, C. Ballesteros, Nanotechnology 16, S197 (2005)

A. Kling, J.C. Soares, A.C. Prieto, J. Jiménez, A. Rodríguez, J. Sangrador, T. Rodríguez, Nucl. Instr. Meth. Phys. Res. B 240, 405 (2005)

A. Kling, M.I. Ortiz, A.C. Prieto, A. Rodríguez, T. Rodríguez, J. Jiménez, C. Ballesteros, J.C. Soares, Nucl. Instr. Meth. Phys. Res. B 249, 306 (2006)

M.I. Ortiz, J. Sangrador, A. Rodríguez, T. Rodríguez, A. Kling, N. Franco, N.P. Barradas, C. Ballesteros, Phys. Stat. Sol. (a) 203, $1284(2006)$

A.V. Kolobov, J.Appl. Phys. 87, 2926 (2000)

H. Campbell, P.M. Fauchet, Solid State Commun. 58, 739 (1986)

J. Olivares, P. Martín, A. Rodríguez, J. Sangrador, J. Jiménez, T. Rodríguez, Thin Solid Films 358, 56 (2000)

J. Olivares, J. Sangrador, A. Rodríguez, T. Rodríguez, J. Electrochem. Soc. 148, C685 (2001)

M. Zacharias, J. Blasing, P. Veit, L. Tsybeskov, K. Hirschman, P.M. Fauchet, Appl. Phys. Lett. 74, 2614 (1999)

Z.H. Lu, D.J. Lockwood, J.M. Baribeau, Solid-State Electron. 40, 197 (1996)

J.C. Tsang, P.M. Mooney, F. Dacol, J.O. Chu, J. Appl. Phys. 75, 8098 (1994)

D.E. Aspnes, A.A. Studna, Phys. Rev. B 27, 985 (1983)

K.L. Teo, S.H. Kwok, P.Y. Yu, S. Guha, Phys. Rev. B 62, 1584 (2000)

P. Martin, A. Torres, J. Jiménez, A. Rodriguez, J. Sangrador, T. Rodríguez, J. Appl. Phys. 96, 155 (2004) 